\title{
DETECCIÓN DE FALLAS EN MOTORES DE COMBUSTIÓN MEDIANTE INDICADORES DE TEMPERATURA Y PRESIÓN DE INYECCIÓN
}

\author{
Detection of Faults in Combustion \\ EngINES Through Indicators OF \\ Temperature and Injection Pressure
}

\author{
Edilberto Antonio Llanes-Cedeño ${ }^{1, *}$, Yans Guardia-Puebla ${ }^{2}$, Alain de la \\ Rosa-Andino $^{2}$, Santiago Cevallos-Carvajal ${ }^{3}$, Juan Carlos Rocha-Hoyos ${ }^{1}$
}

\section{Resumen}

El presente trabajo tiene como objetivo proponer indicadores para la detección temprana de fallas en los motores combustión interna de los grupos electrógenos de fueloil a partir de la presión de inyección y temperatura de la cámara de combustión. Como caso de estudio fueron evaluados los grupos de generación de la Empresa de Mantenimiento a los Grupos Electrógenos Fueloil (EMGEF) en la provincia cubana de Granma. Para el experimento se utilizó un diseño multifactorial usando como factores principales los 16 motores, los 9 cilindros de cada motor y un tiempo de trabajo de 3 años. El estudio demostró que la presión y la temperatura son indicadores significativos en las fallas de los motores, además de que el número de fallas detectadas por temperatura fueron más significativas que las reportadas por la presión de inyección. Se concluye que las altas temperaturas en los cilindros generalmente están relacionadas con un alto índice de gases y un deficiente estado de los inyectores. Las diferencias entre las presiones están relacionadas con la baja hermeticidad y el estado técnico de los elementos del sistema de alimentación.

Palabras clave: fallas, presión, inyección, temperatura, cámara de combustión, mantenimiento, grupos electrógenos.

\section{Abstract}

The present work aims at proposing indicators for early detection of faults in the fuel-oil generator sets of internal combustion engines, using the injection pressure and temperature of the combustion chamber. As a case study, the generation groups of the Maintenance Company of Fuel-Oil Generating Sets (EMGEF), in the Cuban province of Granma, were evaluated. A multifactorial design was used for the experiment, using 16 engines as main factors, the 9 cylinders of each engine, and a working time of 3 years. The study demonstrated that pressure and temperature are significant indicators of engine failure, and that the number of detected faults from temperature were more significant than those reported from injection pressure. It is concluded that high temperatures in the cylinders are generally related to a high index of gases, and a poor state of the injectors. The differences between the pressures are related to low hermetism, and the technical state of the elements of the feeding system.

Keywords: failure, pressure, injection, temperature combustion chamber, maintenance, generator sets.

\footnotetext{
$\overline{1, *}$ Grupo de Investigación de emisiones, Facultad de Arquitectura e Ingeniería, Carrera de Mecánica, Universidad Internacional SEK, Quito-Ecuador. Autor para correspondencia antonio.llanes@uisek.edu.ec

(D) http://orcid.org/0000-0001-6739-7661, (D) http://orcid.org/0000-0003-0660-7199,

${ }^{2}$ Facultad de Ingeniería, Carrera de Mecánica, Universidad de Granma-Cuba. (D) http://orcid.org/0000-0002-1347-0963, (D) http://orcid.org/0000-0001-6593-8583.

${ }^{3}$ Departamento de Energía y Mecánica, Universidad de las Fuerzas Armadas, Ecuador.

(1) http://orcid.org/0000-0001-7998-4978.
}

Recibido: 17-03-2019, aprobado tras revisión: 31-05-2019

Forma sugerida de citación: Llanes-Cedeño, E. A.; Guardia-Puebla, Y.; De la Rosa-Andino, A.; Cevallos-Carvajal, S. y Rocha-Hoyos, J. C. (2019). «Detección de fallas en motores de combustión mediante indicadores de temperatura y presión de inyección». InGENIUs. N. ${ }^{\circ}$ 22, (julio-diciembre). pp. 38-46. DOI: https://doi.org/10.17163/ings.n22.2019.04. 


\section{Introducción}

La generación de electricidad es un campo prioritario para los gobiernos, pues de ello depende en gran medida la calidad de vida de sus ciudadanos. Una de las alternativas para garantizar dicho objetivo son los grupos electrógenos (GE), los cuales se emplean de forma intensiva o como de apoyo. Estos son continuamente mejorados para garantizar su eficiencia y eficacia [1].

Los GE, como todos los equipos de generación distribuida, presentan varias ventajas y desventajas en su funcionamiento, debido a los parámetros del fabricante y de los generadores que lo componen. Sus principales ventajas radican en que ayudan a la conservación del ambiente y son un pilar importante en aumentar la generación eléctrica en los horarios picos cuando se incrementa la demanda, ayudando a la descongestión de los sistemas de transporte de energía. La principal desventaja de este sistema es que pueden provocar fluctuaciones de voltaje que afecten a otros grupos electrógenos cercanos. Además, requiere un sistema de adquisición de datos más complejo que cualquier otro equipo en cuestión, por la tecnología con la que se trabaja en estos casos $[2,3]$.

Numerosos trabajos investigativos se han llevado a cabo con el objetivo de proponer técnicas para la detección y diagnóstico temprano de fallas en los motores de combustión interna. Según Mendonça [4], mediante la medición de la tensión y corriente del estator del generador, se determinan defectos incipientes en los componentes de los generadores impulsados por motores de combustión interna, como son fallas en la válvula de admisión y en la compresión por desgaste de los segmentos del pistón. El estudio realizado por $\mathrm{Xi}, \mathrm{Li}$, Tian, y Duan [5] muestra la posibilidad de detección de fallas en motores de combustión marino a partir del análisis en la frecuencia de las vibraciones.

Por su parte, el estudio realizado por Lee, Cha, Ko, Park, y Jung [6] trata de aplicaciones de detección de fallas y algoritmos de diagnóstico basados en el filtro de Kalman y el método del factor de falla aplicado a un motor cohete propulsor líquido de ciclo abierto en estado estable, permitiendo determinar donde se produce la falla, ya sea en un sensor o componente interno. En estudio realizado por Czech, Wojnar, Burdzik, Konieczny y Warczek [7] describieron los resultados sobre daños en los elementos mecánicos del motor de combustión automotriz (válvula de escape, inyectores, junta de culata), utilizando señales de vibración y redes neuronales artificiales. Los resultados confirmaron la posibilidad de diagnosticar el estado técnico de los componentes del motor del automóvil mientras el motor está en funcionamiento.

Flett y Bone [8] desarrollaron un sistema de detección y diagnóstico de fallas para el tren de válvulas del motor de combustión interna, este método estaba basado en la vibración. Por su parte Trujillo et al. [9] proponen una metodología que combina procedimientos numéricos y experimentales mediante simulaciones por elementos finitos, uso de termopares y termografía infrarroja para la estimación de la temperatura media de la superficie interna del cilindro en un motor diésel de inyección directa de cuatro tiempos refrigerado por aire, mejorándose la toma de datos y evitándose tomar medidas desde el interior de la cámara de combustión, lo que reduce la complejidad de los experimentos a la hora de realizar el diagnóstico.

Durante el cumplimiento normal de las funciones, los GE están sometidos a diferentes solicitudes que ocasionan su deterioro y la consiguiente reducción de la capacidad de generación eléctrica. El deterioro comprende todas las formas de desgaste y desgarradura ocasionadas por fenómenos tales como: fatiga, corrosión, abrasión, erosión y degradación. Estas fallas, es posible que puedan estar provocadas por el efecto de la presión de inyección y la temperatura acumulada dentro de cada cilindro del motor. Por tanto, el objetivo de esta investigación es proponer indicadores para la detección temprana de fallas en los motores combustión interna de los grupos electrógenos de fueloil a partir de la presión de inyección y temperatura de la cámara de combustión.

\section{Materiales y métodos}

Para el estudio de caso se toma como referencia la Unidad Empresarial de Base de $110 \mathrm{kV}$ perteneciente a la Empresa Eléctrica Granma-Cuba, la misma que está compuesta por dieciséis grupos electrógenos que funcionan con fueloil.

\subsection{Extensión de la investigación}

La investigación abarcó un período de 36 meses (lapso comprendido entre enero de 2015 hasta diciembre de 2017). La recopilación de datos e información de valor fue consultada en los siguientes documentos: i) carta de régimen tecnológica para HHI 1,7 MW PPS (código: UJ-IG-0304); ii) control de disponibilidad GDECU (Código: UJ-IG 0105); iii) libro de control de defecto (Código: UJ-MP 0200.A5); iv) libro de incidencias de operación (Código: UJ-MG 0200.A8); v) control operativo de fallas. Se realizaron consultas directas a operadores y especialistas encargados de la explotación de los GE, extrayendo las particularidades de la funcionabilidad de dichos equipos.

\subsection{Título principal grupos electrógenos (GE)}

Los GE de la estación cuentan con 16 motores Hyundai de 1,7 MW. En la Tabla 1 se muestra un resumen de las características de los motores. Estas máquinas utilizan fueloil como combustible, el cual es una fracción del petróleo que se obtiene como residuo luego de la 
destilación. Es el combustible más pesado de los que se puede destilar a presión atmosférica. Se usa como combustible para plantas de energía eléctrica, calderas y hornos.

Tabla 1. Características de los motores HYUNDAY $1,7 \mathrm{MW}$

\begin{tabular}{cc}
\hline Tipo de motor & $\begin{array}{c}\text { HYUNDAY } \\
\mathbf{1 , 7} \text { MW }\end{array}$ \\
\hline Cantidad de cilindros & 9 \\
Velocidad de rotación (min-1) & 1000 \\
Diámetro de los cilindros (mm) & 210 \\
Potencia por cada cilindro $(\mathrm{kW})$ & 200 \\
Carrera de los pistones $(\mathrm{mm})$ & 320 \\
\hline
\end{tabular}

\subsection{Control, análisis y criterios de fallas}

En este apartado se especifican las consideraciones asumidas durante la experimentación.

\subsubsection{Control}

Se recopiló toda la información de las fallas mediante el registro operativo de fallas y el libro de control de fallas. En el mismo se tuvo en cuenta lo siguiente: i) identificación de las averías y fallos repetitivos, clasificación por tipo de equipo o sistema; ii) identificación de las causas de los fallos; iii) recolección de otras informaciones: costo de reparación, tiempo medio entre fallas (TMEF) y tiempo medio por fallas (TMPR); iv) identificación de las acciones correctivas utilizadas anteriormente.

\subsubsection{Criterio de fallas}

Con el fin de definir la gravedad de la falla, se tuvieron en cuenta los indicadores de gravedad referidos por Aguilar-Otero, Torres-Arcique, Magaña-Jiménez [10], Moubray [11] y Scarpatti [12]. Estos indicadores recogen principalmente la experiencia especializada obtenida en el análisis, tratamiento y consecuencias de las fallas que típicamente afectan los esquemas de control y protección.

\subsubsection{Diseño experimental}

Los resultados de los análisis y los gráficos fueron obtenidos a partir del paquete estadístico STATGRAPHICS Centurion XV (Trial version 15, StatPoint Inc., USA). Para el diseño experimental fue utilizado un diseño factorial, en el cual se estudian los efectos de los factores cuantitativos (Ecuación 1). El modelo matemático del diseño se definió como:

$$
\begin{array}{r}
y_{i j k}=\mu+\tau_{i}+\beta_{j}+\gamma_{k}+(\tau \beta)_{i j}+(\beta \gamma)_{j k}+ \\
+(\tau \beta \gamma)_{i j k}+\epsilon_{i j k}\left\{\begin{array}{l}
i=1,2, \ldots, a \\
j=1,2, \ldots, b \\
k=1,2, \ldots, c
\end{array}\right.
\end{array}
$$

Donde las variables $\tau, \beta, \gamma$ representan los factores motores, cilindro y tiempo de trabajo, así como sus interacciones. Para el análisis de los niveles de los factores se utilizó la metodología del análisis de varianza (Anova), donde se calculó un valor F para un valor de probabilidad determinado $(0,05)$ y se comparó con un valor tabulado, para así poder determinar diferencias significativas entre los niveles de los factores [13-15]. Estos fueron definidos así: el factor motor fue fijado a 15 motores, con 9 cilindros en cada motor, y el tiempo de trabajo se evaluó en 3 años (2015-2017).

\subsubsection{Instrumentos de medición}

Para la obtención de los valores de presión de inyección y la temperatura de la cámara de combustión acumulada en cada cilindro fue utilizado un Leutert modelo DPI-2 (Digital Pressure Indicator), este instrumento permite determinar la presión y temperatura al ingreso. El sistema DPI incluye un sensor, con el cual se determina la presión de inyección en los motores diésel. Los datos obtenidos fueron procesados y evaluados con el software del dispositivo, versión 3.24 para Windows®.

\section{Resultados y discusión}

En este apartado se exponen y discuten los resultados obtenidos durante la experimentación.

\subsection{Análisis de la temperatura en la cámara de combustión (cc) de los motores}

La Tabla 2 muestra las mediciones de temperatura de las cámaras combustión de los 15 motores bajo estudio. Los factores principales, motores, tiempo de trabajo y su combinación, fueron altamente significativos a un $95 \%$ de probabilidad, ya que los p-valores del Anova no superaron el límite de 0,05. Estos resultados indican que existe una alta variabilidad en la entrega de energía del emplazamiento, debido a que no es eficiente el trabajo realizado por los motores, lo cual influye en la capacidad de entrega de energía a la red nacional. También, un factor que ha influido significativamente es el tiempo de operación realizado por algunos motores, ya que esto depende de la demanda eléctrica que se requiera. La orden de trabajo y la cantidad de energía que se necesita, según la demanda, se obtienen de la central de generación. Esta orden de trabajo indica la cantidad de motores y el porcentaje de funcionamiento que se requiere para suplir esa demanda. Esta situación también genera una 
variabilidad en el tiempo de trabajo del complejo de motores, y por tanto una ineficiencia en el manejo de la tecnología. Otro aspecto importante que influye es operar los motores en parámetros inferiores al considerado como óptimo (inferiores al $70 \%$ de su capacidad eficiente de generación).

El Anova (Tabla 2) también indicó diferencias significativas en la interacción de los factores motores y tiempo de trabajo. La Figura 1 muestra la interacción tiempo de trabajo y motores en función de la temperatura de las cámaras de combustión. No existe una tendencia al incremento de la temperatura en cada motor individual en los meses de verano (mayo a octubre), o una reducción de temperatura en los meses de invierno (noviembre a abril), pues no hay una tendencia definida. Esta fluctuación en los valores de temperatura se debe al mantenimiento realizado en cada motor y la variación de las propiedades físicas del combustible, dependiendo del lote de procedencia y del proveedor [16]. Este último aspecto es muy importante pues cambia los parámetros fisicoquímicos del combustible, tales como: viscosidad, densidad, poder calórico, porcentaje de carbón, asfaltenos, sedimentos, entre otros [17].

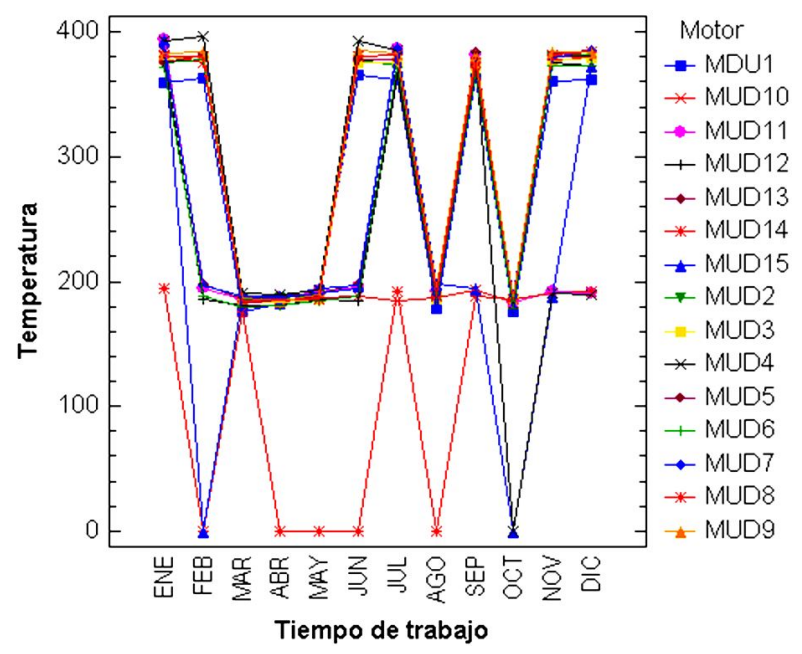

Figura 1. Interacción tiempo de trabajo y motores en función de la temperatura.

Tabla 2. Tabla Anova para temperatura de las cámaras de combustión

\begin{tabular}{|c|c|c|c|c|c|}
\hline Fuente & $\mathbf{S S}^{\mathbf{a}}$ & $\mathbf{G L}^{\mathbf{b}}$ & $\mathrm{CM}^{\mathrm{c}}$ & F-Ratio $^{d}$ & p-valor \\
\hline MCI & $7,44 \cdot 10^{6}$ & 14 & $5,3.10^{5}$ & 24,66 & $0,0000^{\mathrm{e}}$ \\
\hline Cilindro & $3,92.10^{4}$ & 8 & 4906,88 & 0,23 & 0,9860 \\
\hline Tiempo de trabajo & $2,03.10^{7}$ & 11 & $1,85.10^{6}$ & 85,68 & $0,0000^{\mathrm{e}}$ \\
\hline MCI*Cilindro & $2,41 \cdot 10^{5}$ & 112 & 2157,18 & 0,10 & 1,0000 \\
\hline MCI*Tiempo de trabajo & $1,06.10^{7}$ & 154 & $6,9.10^{4}$ & 3,18 & $0,0000^{\mathrm{e}}$ \\
\hline Cilindro*Tiempo de trabajo & $2,1.10^{4}$ & 88 & 238,64 & 0,01 & 1,0000 \\
\hline RESIDUOS & $6,18 \cdot 10^{7}$ & 2865 & $2,15 \cdot 10^{4}$ & & \\
\hline TOTAL (CORREGIDO) & $1,0.10^{8}$ & 3252 & & & \\
\hline
\end{tabular}

Nota: ${ }^{a}$ SC. Suma de cuadrados, ${ }^{b}$ GL. Grados de libertad, ${ }^{c}$ CM. Cuadrados medios,

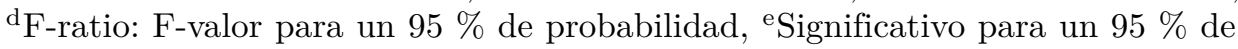
probabilidad

En la Tabla 3 se observa la comparación de medias de temperatura en las cámaras de combustión, y grupos homogéneos entre los niveles de los factores motor y tiempo de trabajo. En la misma se observa que no existe una distribución uniforme en la temperatura observada en los $15 \mathrm{MCI}$ del parque generador, indicando un amplio rango de temperatura promedio que oscila entre $109-302{ }^{\circ} \mathrm{C}$. Este rango de valores está por debajo del valor óptimo que es de $320{ }^{\circ} \mathrm{C}$. Por otro lado, se observó una tendencia a disminuir la temperatura global promedio de los MCI en los meses comprendidos entre noviembre-marzo, correspondiendo con lo meses más fríos, estos resultados coinciden con los referidos por $[1,10]$.

\subsection{Análisis de la presión de inyección en los motores}

La Tabla 4 muestra el análisis de varianza para la presión de inyección en función de los factores antes mencionados. Los factores principales MCI y tiempo de trabajo siguieron siendo significativos, indicando que existen diferencias de presión de inyección entre los 15 motores. La variabilidad entre los MCI se debe principalmente a las paradas del funcionamiento, que pueden estar relacionadas con el mantenimiento, revisión técnica, averías, o sencillamente porque no hay un aumento en la demanda y la central energética determina poner fuera de servicio algunos motores. 
Tabla 3. Comparación de medias de temperatura en las cámaras de combustión y entre los niveles de los factores motor y tiempo de trabajo

\begin{tabular}{|c|c|c|c|c|c|}
\hline Motor & Media $\left({ }^{\circ} \mathrm{C}\right)$ & $\begin{array}{c}\text { Grupos } \\
\text { homogéneos }\end{array}$ & $\begin{array}{c}\text { Tiempo } \\
\text { de trabajo }\end{array}$ & Media $\left({ }^{\circ} \mathbf{C}\right)$ & $\begin{array}{c}\text { Grupos } \\
\text { homogéneos }\end{array}$ \\
\hline MDU1 & $286,6 \pm 9,8$ & XXXX & ENE & $172,9 \pm 8,9$ & $\mathrm{X}$ \\
\hline MUD2 & $297,9 \pm 9,9$ & XXX & FEB & $176,5 \pm 8,9$ & $\mathrm{X}$ \\
\hline MUD3 & $296,8 \pm 9,9$ & XXX & MAR & $177,1 \pm 8,9$ & $\mathrm{X}$ \\
\hline MUD4 & $258,0 \pm 9,9$ & XX & $\mathrm{ABR}$ & $369,0 \pm 8,9$ & X \\
\hline MUD5 & $298,0 \pm 9,9$ & XXX & MAY & $337,9 \pm 8,9$ & XX \\
\hline MUD6 & $261,7 \pm 9,9$ & XXX & JUN & $353,0 \pm 8,9$ & XX \\
\hline MUD7 & $271,4 \pm 9,9$ & XXX & JUL & $266,6 \pm 8,9$ & X \\
\hline MUD8 & $299,4 \pm 9,9$ & XX & AGO & $266,8 \pm 8,9$ & $\mathrm{X}$ \\
\hline MUD9 & $302,6 \pm 9,9$ & X & SEP & $340,8 \pm 8,9$ & XX \\
\hline MUD10 & $250,2 \pm 9,9$ & XX & OCT & $327,4 \pm 8,9$ & X \\
\hline MUD11 & $239,6 \pm 9,9$ & $\mathrm{X}$ & NOV & $159,8 \pm 8,9$ & $\mathrm{X}$ \\
\hline MUD12 & $262,2 \pm 9,9$ & XXX & DIC & $183,8 \pm 8,9$ & $\mathrm{X}$ \\
\hline MUD13 & $271,9 \pm 9,9$ & XXXX & & & \\
\hline MUD14 & $109,9 \pm 9,9$ & $\mathrm{X}$ & & & \\
\hline MUD15 & $208,4 \pm 9,9$ & $\mathrm{X}$ & & & \\
\hline
\end{tabular}

El factor tiempo de trabajo está también muy relacionado con la variabilidad de la presión de inyección, ya que a medida que incrementa el tiempo de operación de un motor, se incrementa el desgaste de las piezas, siendo agravado el sistema de alimentación por la variación de la calidad del combustible [18].

No se detectaron diferencias significativas entre los cilindros de los motores, indicando que la presión de inyección del combustible hacia el interior de la cámara de combustión es constante.
En la Figura 2 se observa que los valores de presión de inyección global promedio del parque generador fueron prácticamente constantes en la mayoría de los meses. Según el tiempo de trabajo, solo se observó una divergencia en los meses de abril y mayo. De igual manera, los motores MDU1, MDU2 y MDU9 mostraron incrementos de presión en comparación con el resto de los motores. Estos incrementos pueden estar provocados por la falta de relación entre ángulo de la cremallera y la cantidad de combustible de entrada y tupición de las toberas o en los filtros de combustibles.

Tabla 4. Análisis de varianza para presión de inyección

\begin{tabular}{cccccc}
\hline Fuente & $\mathbf{S S}^{\mathbf{a}}$ & $\mathbf{G L}^{\mathbf{b}}$ & $\mathbf{C M}^{\mathbf{c}}$ & F-Ratio $^{\mathbf{d}}$ & p-valor \\
\hline Motor & 32558,6 & 14 & 2325,6 & 2,19 & $0,0065^{\mathrm{e}}$ \\
Cilindro & 5755,2 & 8 & 719,4 & 0,68 & 0,7119 \\
Tiempo & 28061,5 & 11 & 2551,1 & 2,4 & $0,0058^{\mathrm{e}}$ \\
de trabajo & $2,06 \mathrm{E}+06$ & 1943 & 1062 & & \\
RESIDUOS & 2,062 & & & \\
TOTAL & $2,13 \mathrm{E}+06$ & 1976 & & & \\
(CORREGIDO) & & & & &
\end{tabular}

${ }^{\mathrm{a}} \mathrm{SC}$. Suma de cuadrados, ${ }^{\mathrm{b}}$ GL. Grados de libertad, ${ }^{\mathrm{c}} \mathrm{CM}$. Cuadrados medios, ${ }^{\mathrm{d}}$ F-ratio: F-valor para un $95 \%$ de probabilidad, ${ }^{\text {eSignificativo }}$ para un $95 \%$ de probabilidad. 

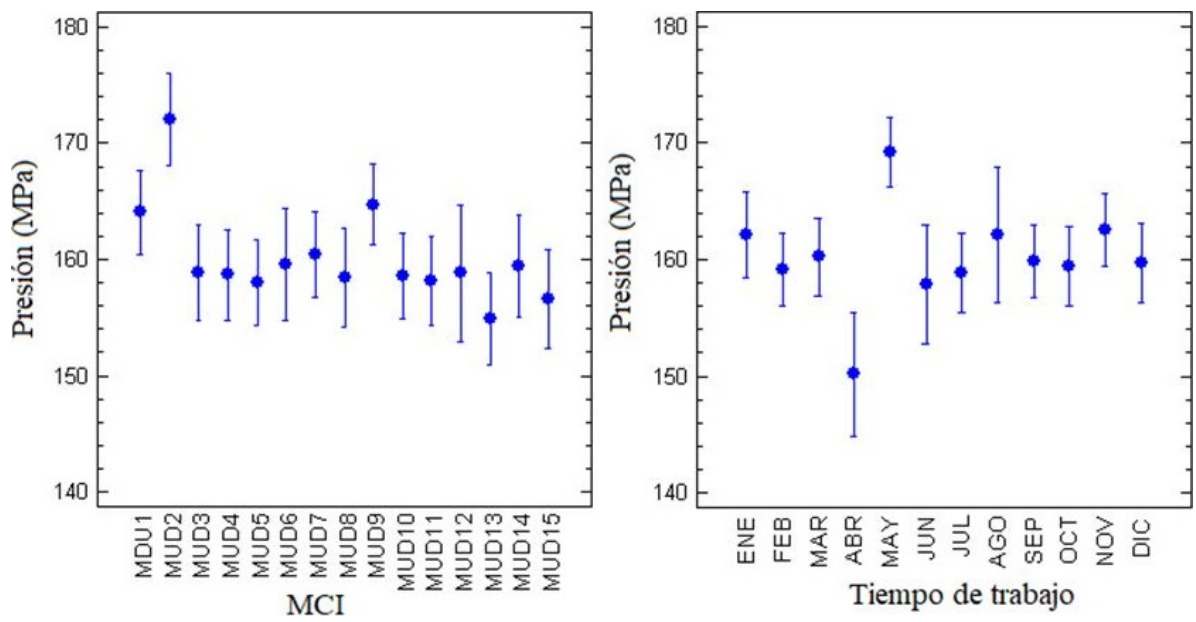

Figura 2. Representación de los rangos de medias de presión por MCI y tiempo de trabajo.

\subsection{Análisis de las fallas por presión de inyección y temperatura en la cámara de combustión}

Como se observa en la Figura 3a, las fallas en el año 2015 estuvieron influenciadas por las altas temperaturas alcanzadas por los MCI del parque generador. Los MCI MDU 15, 9, 11 y 8, en ese orden, fueron los afectados por las altas temperaturas. Esta situación estuvo influenciada por varios factores, los cuales afectaron la capacidad de generación eléctrica del emplazamiento, dentro de estos se destacan el tiempo de operación y la calidad del combustible. Un comportamiento similar se observó en el año 2016 (ver Figura 3b), donde las fallas por temperatura arrojaron valores superiores a las fallas por presión. Sin embargo, en el año 2017 (ver Figura 3c) se aprecia una disminución o descenso en el número de fallas observadas. Pues solamente, el MCI MDU 8 fue el que arrojó mayores valores de fallas. El resto de los MCI del emplazamiento mostraron valores inferiores. En comparación con los años anteriores, este fue el año de menor incidencia, demostrando una mejora en la operación y eficiencia del emplazamiento, debido a un mejor conocimiento y experiencia adquirida por los operadores, así como un avance en el mantenimiento de los equipos.

En la Figura 4 se aprecia cómo se redujo el número de fallas por los efectos de la presión de inyección y temperatura en la cámara de combustión, en algunos MCI del emplazamiento en el año 2018. Esto se debe, a que en este período hubo un mejor manejo de la tecnología. Por lo tanto, no se produjeron anomalías en el funcionamiento del emplazamiento y entrega de energía a la red eléctrica nacional. Aunque se reportaron fallas en algunos MCI como MDU-2, MDU-4, MDU8, MDU-11, MDU-13 y MDU-15, siendo los motores MDU-2 y MDU-13 los de más incidencia con 6 fallas en el período. Estos resultados indican un aumento en la eficiencia de trabajo del grupo electrógeno por la reducción del número de fallas. No obstante, aunque se redujo el número de fallas, se debe trabajar en optimizar la generación del emplazamiento, enfocándose en mejorar la eficiencia de los métodos de operación y decisiones directivas [19].

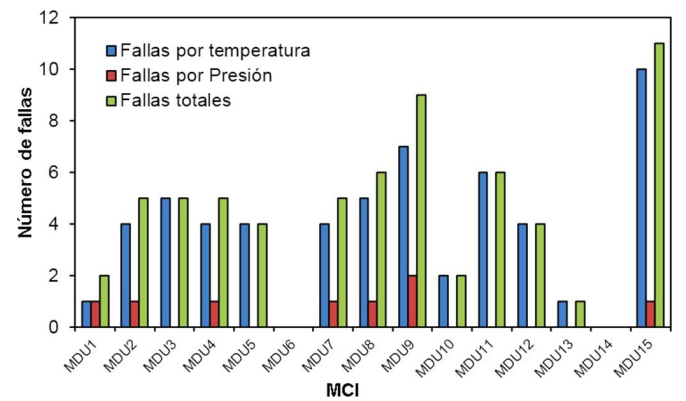

a)

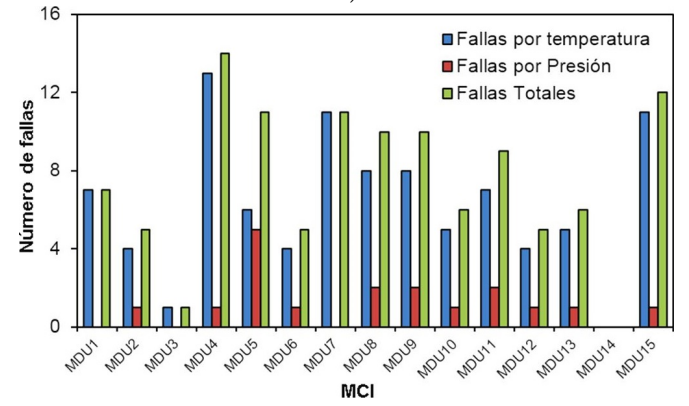

b)

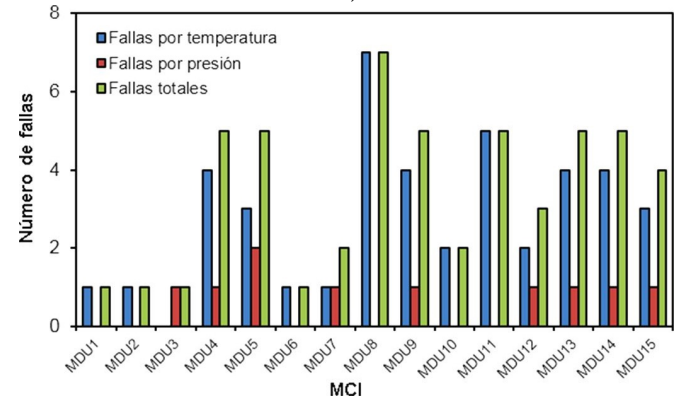

c)

Figura 3. Resumen de fallas por presión y temperatura: a) año 2015; b) año 2016; c) año 2017. 


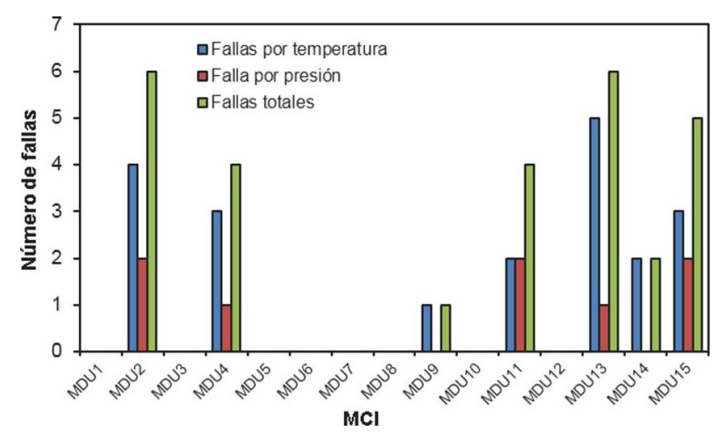

Figura 4. Resumen de fallas por presión y temperatura en el año 2018.

En la Figura 5 se observan las fallas totales provocadas por la presión de inyección y la temperatura en la cámara de combustión, en el período comprendido entre los años 2015-2017.

La mayor falla detectada en el año 2015 (Figura 5a) fue por alta temperatura (82 fallas), la cual está directamente relacionada con el índice del gobernador (55 fallas). El «gobernador» es el encargado de dar el ángulo de apertura de la cremallera, en otras palabras, el acelerador. Cuando el índice es alto indica un aumento en la aceleración del motor, por lo que en un período prolongado de trabajo provoca altas temperaturas y presión en los cilindros [20-22]. Un alto valor de índice de gobernador, y en conjunto con un inadecuado funcionamiento del inyector, puede provocar altas temperaturas en los cilindros, porque permite el control del índice de cremalleras y la entrada del combustible [23]. Al trabajar los inyectores de forma ineficiente, se incrementa la entrada de combustible al interior del cilindro, y se eleva la temperatura por el exceso de combustible en la cámara de combustión [2]. Este fenómeno provoca también la generación de gases residuales de color negro [24].

La tercera falla más detectada fueron los salideros de combustible (23 fallas). Este fenómeno está directamente relacionado con un mal sellaje en el sistema de alimentación, producto de un inadecuado mantenimiento y revisión de los MCI. No obstante, en el año 2016 se corrigieron varias de las fallas observadas en el 2015 (Figura 5b). No se detectaron fallas por sellaje en el sistema de alimentación, se redujo en un $72 \%$ la cantidad de fallas en el índice del gobernador, pero se mantuvieron elevadas las fallas por altas temperaturas (88 fallas); los otros indicadores reportaron un rango de fallas entre 5 - 20. En el año 2017 (Figura 5c) se redujo la cantidad de fallas por alta temperaturas en los cilindros (64 fallas), pero se incrementaron en el índice del gobernador (44 fallas) y la baja hermeticidad (7 fallas).

En la Figura 6 se observa el comportamiento de los MCI en el año 2018, siendo las altas temperaturas (21 fallas) las de mayor incidencia, aunque se observó una tendencia a la disminución de las fallas con respecto a los años anteriores.

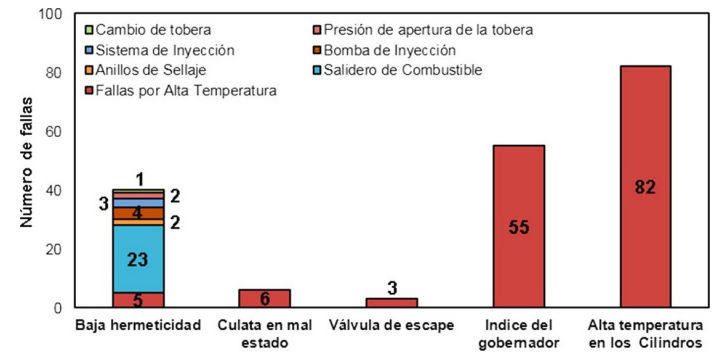

a)

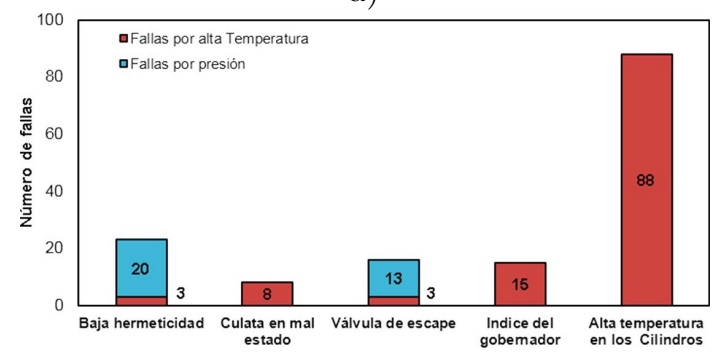

b)

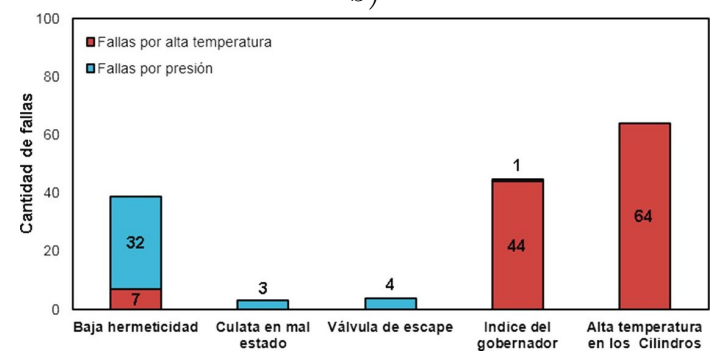

c)

Figura 5. Fallas provocadas por presión y temperatura más significativas y sus efectos en los MCI 9H21/32: a) año 2015 ; b) año 2016; c) año 2017.

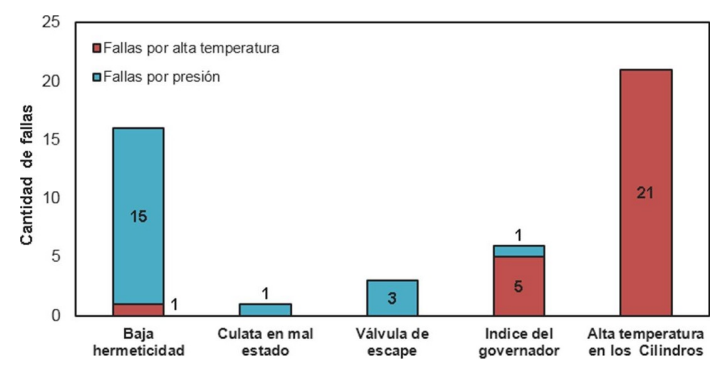

Figura 6. Fallas provocadas por presión de inyección y temperatura en la cámara de combustión, más significativas en el año 2018.

\section{Conclusiones}

El análisis demostró que la presión de inyección y la temperatura en las cámaras de combustión son indicadores influyentes en la determinación de las fallas de los MCI Hyundai de 1,7 MW.

El número de fallas detectadas por temperatura en las cámaras de combustión son significativas en comparación con las reportadas por la presión de inyección. 
Las altas temperaturas en ellas, generalmente están relacionadas con un alto índice de gobernador y un deficiente estado de los inyectores.

La interacción de los factores $M C I^{*}$ tiempo de trabajo resultó estadísticamente significativa en el análisis de la temperatura de la cámara de combustión, provocando ineficiencia en el funcionamiento de los MCI y en la entrega de energía del emplazamiento.

\section{Referencias}

[1] M. C. Fernández, M. F. Fernández, R. D. Fuentes, and A. C. Montiel, "Calidad de la energía y generación distribuida en Cuba," Revista Cubana de Ingeniería, vol. 1, no. 3, pp. 41-50, 2010. [Online]. Available: https://doi.org/10.1234/rci.v1i3.28

[2] J. G. Boza, "Estudio de estabilidad en un sistema eléctrico con grupos electrógenos," Revista de Ingeniería Energética, vol. 28, no. 2, pp. 26-28, 2007. [Online]. Available: http://bit.ly/2F1S6BD

[3] J. F. Quintana Tamayo, F. Martínez Pérez, Y. G. Vázquez Jorge, and J. Ramírez Arzuaga, "Estudio de factibilidad para optimizar frecuencia de reemplazo del lubricante, en grupos electrógenos," Revista Ciencias Técnicas Agropecuarias, vol. 3, no. 23, pp. 60-68, 09, 2014. [Online]. Available: http://bit.ly/31iGFz3

[4] P. Mendonça, E. Bonaldi, L. de Oliveira, G. Lambert-Torres, J. B. da Silva, L. B. da Silva, C. Salomon, W. Santana, and A. Shinohara, "Detection and modelling of incipient failures in internal combustion engine driven generators using electrical signature analysis," Electric Power Systems Research, vol. 149, pp. 30-45, 2017. [Online]. Available: https://doi.org/10.1016/j.epsr.2017.04.007

[5] W. Xi, Z. Li, Z. Tian, and Z. Duan, "A feature extraction and visualization method for fault detection of marine diesel engines," Measurement, vol. 116, pp. 429-437, 2018. [Online]. Available: https: //doi.org/10.1016/j.measurement.2017.11.035

[6] K. Lee, J. Cha, S. Ko, S.-Y. Park, and E. Jung, "Fault detection and diagnosis algorithms for an open-cycle liquid propellant rocket engine using the kalman filter and fault factor methods," Acta Astronautica, vol. 150, pp. 15-27, 2018. [Online]. Available: https://doi.org/10.1016/j.actaastro.2018.03.001

[7] P. Czech, G. Wojnar, R. Burdzik, L. Konieczny, and J. Warczek, "Application of the discrete wavelet transform and probabilistic neural networks in ic engine fault diagnostics," Journal of
Vibroengineering, vol. 16, pp. 1619-1639, 2014. [Online]. Available: http://bit.ly/2XBwbIF

[8] J. Flett and G. M. Bone, "Fault detection and diagnosis of diesel engine valve trains," Mechanical Systems and Signal Processing, vol. 72-73, pp. 316-327, 2016. [Online]. Available: https://doi.org/10.1016/j.ymssp.2015.10.024

[9] E. Carvajal Trujillo, F. J. Jiménez-Espadafor, J. A. Becerra Villanueva, and M. Torres García, "Methodology for the estimation of cylinder inner surface temperature in an air-cooled engine," Applied Thermal Engineering, vol. 31, no. 8, pp. 1474-1481, 2011. [Online]. Available: https: //doi.org/10.1016/j.applthermaleng.2011.01.025

[10] J. R. Aguilar-Otero, R. Torres-Arcique, , and D. Magaña Jiménez, "Análisis de modos de falla, efectos y criticidad (AMFEC) para la planeación del mantenimiento empleando criterios de riesgo y confiabilidad," Tecnología, Ciencia, Educación, vol. 25, pp. 15-26, 2010. [Online]. Available: http://bit.ly/2I8mAnx

[11] J. Moubray, Mantenimiento centrado en la confiabilidad. Industrial Press Inc., 2004. [Online]. Available: http://bit.ly/2Izu4yC

[12] F. Scarpatti, "Análisis de modos de fallas y sus efectos," Scarpatti y Asociados. Consultores en Gestión Organizacional, Tech. Rep., 2001. [Online]. Available: http://bit.ly/2F2mprX

[13] D. C. Montgomery, Design and Analysis of Experiment. John Wiley \& Sons, 2008. [Online]. Available: http://bit.ly/2WyxprV

[14] J. C. Rocha-Hoyos, E. A. Llanes-Cedeño, S. CeliOrtega, and D. Peralta-Zurita, Efecto de la mezcla de biodiesel en el rendimiento y la opacidad del motor diesel. Información Tecnológica, 2019.

[15] Y. Guardia-Puebla, J. Márquez-Delgado, V. Sánchez-Girón, E. A. Llanes-Cedeño, J. C. Rocha-Hoyos, and D. B. Peralta-Zurita, "Mejoras a la asignatura diseño estadístico de experimentos para estudiantes de la carrera de Ingeniería Mecánica," Revista Espacios, vol. 39, no. 30, pp. 10-25, 2018. [Online]. Available: http://bit.ly/2KJbs2e

[16] F. Martínez Pérez and A. Barroso Moreno, "Aplicación de la tribología y el análisis de la causa raíz (RCA) en motores de combustión interna," Ingeniería Mecánica, vol. 3, pp. 53-56, 2008. [Online]. Available: http://bit.ly/2IA0ppd

[17] D. Fernández-Fernández and F. Mourdoch-Misa, "Aplicación de técnicas de lubricación predictivas 
en grupos electrógenos," Ingeniería Mecánica, vol. 12 , no. 2, pp. 1-8, 2009. [Online]. Available: http://bit.ly/2F3vbpw

[18] K. Riplová, "Tool of risk management: failure mode and effects analysis and failure modes, effects and criticality analysis," Journal of Information, Control and Management Systems, vol. 5, no. 1, pp. 111-120, 2007. [Online]. Available: http://bit.ly/2KbKm4i

[19] O. Martínez Rodríguez and S. A. Dorrbercker Drake, "Determinación de las prioridades de mantenimiento en turbogeneradores "Elektrosila TBФ-100-3600-T3" a partir del comportamiento de sus fallos," Ingeniería Energética, vol. 33, no. 3, pp. 250-262, 2012. [Online]. Available: http://bit.ly/2Rak4zR

[20] S. Wu and D. Clements-Croome, "Ratio of operating and maintenance costs to initial costs of building services systems," Cost Engineering, vol. 49, no. 12, pp. 30-33, 2007. [Online]. Available: http://bit.ly/2Ialckl

[21] S. You-Cheng, X. Min, G. Yong, C. Yi, S. Lei, and D. Kang-yao, "Effects of injection pressure, exhaust gas recirculation and intake pressure on the cycle-to-cycle variations of HCCI combustion," Journal of the Energy Institute, vol. 89, no. 2, pp. 293-301, 2016. [Online]. Available: https://doi.org/10.1016/j.joei.2015.01.017

[22] A. K. Agarwal, D. K. Srivastava, A. Dhar, R. K. Maurya, P. C. Shukla, and A. P. Singh, "Effect of fuel injection timing and pressure on combustion, emissions and performance characteristics of a single cylinder diesel engine," Fuel, vol. 111, pp. 374-383, 2013. [Online]. Available: https://doi.org/10.1016/j.fuel.2013.03.016

[23] S. Gowthaman and A. Sathiyagnanam, "Effects of charge temperature and fuel injection pressure on hcci engine," Alexandria Engineering Journal, vol. 55, no. 1, pp. 119-125, 2016. [Online]. Available: https://doi.org/10.1016/j.aej.2015.12.025

[24] E. A. Llanes-Cedeño, J. C. Rocha-Hoyos, Y. Guardia-Puebla, L. Tipanluisa, and G. Velastegui, "Análisis comparativo de los gases residuales de la combustión y fallas en motores Hyundai modelo 9h21/32, en estación de generación eléctrica distribuida," INNOVA Research Journal, vol. 2, no. 3, pp. 27-41, 2017. [Online]. Available: http://bit.ly/2R5PE1P 